\title{
Tangle Embeddings and Quandle Cocycle Invariants
}

\author{
Kheira Ameur Mohamed Elhamdadi Tom Rose \\ Masahico Saito* Chad Smudde \\ Department of Mathematics and Statistics \\ University of South Florida
}

\begin{abstract}
To study embeddings of tangles in knots, we use quandle cocycle invariants. Computations are carried out for the tables of knots and tangles, to investigate which tangles may or may not embed in knots in the tables.
\end{abstract}

\section{Introduction}

A tangle is a pair $(B, A)$ where $A$ is a set of properly embedded arcs in a 3-ball $B$. A tangle will have four end points throughout the article, unless otherwise specified. A tangle $T$ is embedded in a link (or a knot) $L$ if there is an embedded ball $B$ in 3-space such that $T$ is equivalent to the pair $(B, B \cap L)$. All maps are assumed to be smooth. Tangles are represented by diagrams in a manner similar to knot diagrams.

Tangle embeddings have been studied by several authors recently. In [11, the determinant was used in relation to evaluations of the Jones polynomial, that have been further investigated in [5, 11, 12]. Topological interpretations were considered in [18, 19]. Tangles were also used to study DNA recombinations [7].

In this article, we present a method of using quandle cocycle invariants as obstructions to embedding tangles in knots, and examine their effectiveness as obstructions by looking at the table of tangles presented in [10] and the knot table in [13]. Quandles are self-distributive sets with additional properties (see below for details), and have been used in the study of knots since 1980s. A cohomology theory of quandles have been developed, and their cocycles have been used as state-sum invariants of knots and knotted surfaces [4]. Quandles are used to investigate tangles also in [6, 17].

\footnotetext{
*Supported in part by NSF Grant DMS \#0301089, \#0603876.
} 
In this paper, we focus on effectiveness of quandle cocycle invariants as obstructions. It will be shown that the invariants often provide effective obstructions when a given tangle has non-trivial colorings by quandles.

The paper is organized as follows. After a review of preliminary material in Section 2, colorings of tangles are defined in Section 3, and the tangles in the table (see Fig. 6) that have non-trivial colorings by Alexander quandles are listed. The main theorem is presented in Section 4, For tangles listed in Section 3, it is examined which tangles may or may not embed in knots in the knot table. In Section [5, embeddings of multiple disjoint copies of tangles are discussed. Part of the results are based on the work in the Ph.D. dissertation by Kheira Ameur [2].

\section{Preliminaries}

\subsection{Tangles and their operations}

The conventions described in this subsection are commonly found in the literature (see for example, [1, 16]).

The four end points of a given tangle diagram $T$ are located at four corners of a circle in a plane at angles $\pi / 4,3 \pi / 4,5 \pi / 4$ and $7 \pi / 4$, when the circle is placed with the origin as its center. These end points are labeled by NE, NW, SW, and SE, respectively, representing North East, etc.

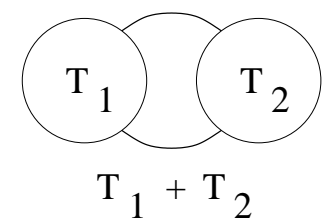

Figure 1: Addition of tangles

The addition $T_{1}+T_{2}$ of two tangles $T_{1}, T_{2}$ is another tangle defined from the original two as depicted in Fig. 1. There are two ways of closing the end points of a tangle, called closures, the numerator $N(T)$ and denominator $D(T)$ of a tangle $T$, defined as depicted in Fig. 2,

There is a family of "trivial" or "rational" tangles, some of which are depicted in Fig. 3. These tangles are obtained from the trivial tangle of two vertical straight arcs by successively twisting end points vertically and horizontally. See again [1] or [16] for more details. 


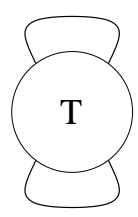

$\mathrm{N}(\mathrm{T})$

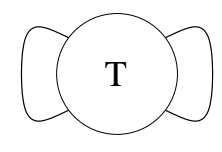

$\mathrm{D}(\mathrm{T})$

Figure 2: Closures (numerator $N(T)$ and denominator $D(T)$ ) of tangles

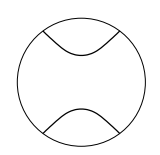

$\mathrm{R}(0)$

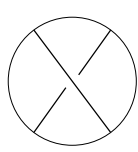

$\mathrm{R}(1)$

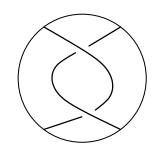

$\mathrm{R}(2)$

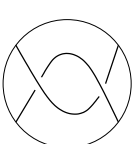

$\mathrm{R}(1,1)$

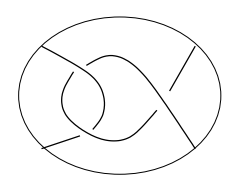

$\mathrm{R}(2,1)$

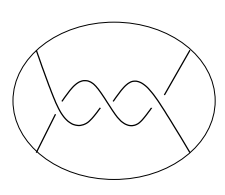

$\mathrm{R}(1,2)$

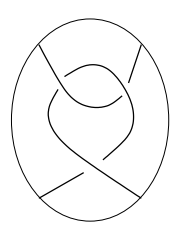

$\mathrm{R}(1,1,1)$

Figure 3: Some rational tangles

In [10, prime tangles (with crossing number at most seven) are classified, and a table of their diagrams is given. The table consists of a single 5 crossing tangle followed by four 6 crossing tangles, and 18 tangles of 7 crossings. Some multiple component tangles were also classified. The tangles are named in a scheme similar to knots by integers with subscripts. Some of the tangles are presented in Fig. 6 ,

\subsection{Quandles, colorings, and cocycle invariants}

A quandle, $X$, is a set with a binary operation $(a, b) \mapsto a * b$ such that

(I) For any $a \in X, a * a=a$,

(II) For any $a, b \in X$, there is a unique $c \in X$ such that $a=c * b$,

(III) For any $a, b, c \in X$, we have $(a * b) * c=(a * c) *(b * c)$.

A rack is a set with a binary operation that satisfies (II) and (III). Racks and quandles have been studied in, for example, [3, 8, 9, 14.

The following are typical examples of quandles. A group $G$ with conju- 


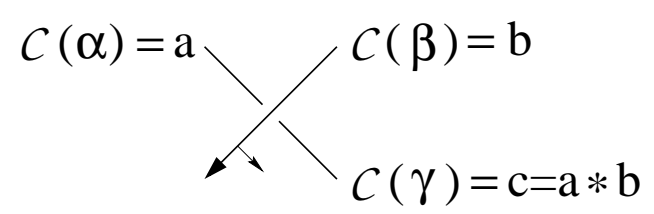

Figure 4: Quandle relation at a crossing

gation as the quandle operation, $a * b=b a b^{-1}$, is a quandle. Any $\mathbb{Z}\left[t, t^{-1}\right]$ module $M$ is a quandle with $a * b=t a+(1-t) b, a, b \in M$, that is called an Alexander quandle. Let $n$ be a positive integer, and for elements $i, j \in \mathbb{Z}_{n}$, define $i * j \equiv 2 j-i(\bmod n)$. Then $*$ defines a quandle structure called the dihedral quandle, $R_{n}$.

Let $X$ be a fixed quandle. Let $K$ be a given oriented classical knot or link diagram, and let $\mathcal{R}$ be the set of (over-)arcs. The normals (normal vectors) are given in such a way that the ordered pair (tangent, normal) agrees with the orientation of the plane, see Fig. 4. A (quandle) coloring $\mathcal{C}$ is a map $\mathcal{C}: \mathcal{R} \rightarrow X$ such that at every crossing, the relation depicted in Fig. 4 holds. Specifically, let $\beta$ be the over-arc at a crossing, and let $\alpha$ and $\gamma$ be the under arcs, such that the normal of the over-arc points from $\alpha$ to $\gamma$, then $\mathcal{C}(\alpha) * \mathcal{C}(\beta)=\mathcal{C}(\gamma)$ holds. The (ordered) colors $(\mathcal{C}(\alpha), \mathcal{C}(\beta))$ are called source colors. Let $\mathrm{Col}_{X}(K)$ denote the set of colorings of a knot diagram $K$ by a quandle $X$.

Let $K$ be a knot diagram on the plane. Let $X$ be a finite quandle and $A$ be an abelian group. Let $\phi: X \times X \rightarrow A$ be a quandle 2-cocycle, which can be regarded as a function satisfying the 2 -cocycle condition

$$
\phi(x, y)-\phi(x, z)+\phi(x * y, z)-\phi(x * z, y * z)=0, \quad \forall x, y, z \in X
$$

and $\phi(x, x)=0, \forall x \in X$. Let $\mathcal{C}$ be a coloring of a given knot diagram $K$ by $X$.

The Boltzmann weight $B(\mathcal{C}, \tau)=B_{\phi}(\mathcal{C}, \tau)$ at a crossing $\tau$ of $K$ is then defined by $B(\mathcal{C}, \tau)=\epsilon(\tau) \phi\left(x_{\tau}, y_{\tau}\right)$, where $\left(x_{\tau}, y_{\tau}\right)$ is the source colors at $\tau$ and $\epsilon(\tau)$ is the sign $( \pm 1)$ of $\tau$. Then the 2-cocycle invariant $\Phi(K)=\Phi_{\phi}(K)$ in a multiset form is defined by

$$
\Phi_{\phi}(K)=\left\{\sum_{\tau} B(\mathcal{C}, \tau) \mid \mathcal{C} \in \operatorname{Col}_{\mathrm{X}}(\mathrm{K})\right\} .
$$

(A multiset is a collection of elements where a single element can be repeated multiple times, such as $\{0,0,1,1,1\}$, which is also denoted by $\left.\left\{\sqcup_{2} 0, \sqcup_{3} 1\right\}\right)$. 


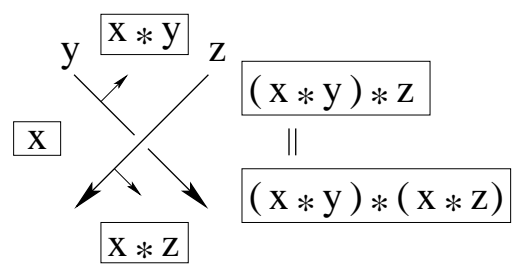

Figure 5: Region colors at a crossing

Let $\theta: X \times X \times X \rightarrow A$ be a quandle 3-cocycle, which can be regarded as a function satisfying

$$
\begin{aligned}
& \theta(x, z, w)-\theta(x, y, w)+\theta(x, y, z)-\theta(x * y, z, w) \\
& \quad+\theta(x * z, y * z, w)-\theta(x * w, y * w, z * w)=0, \quad \forall x, y, z, w \in X,
\end{aligned}
$$

and $\theta(x, x, y)=0=\theta(x, y, y), \forall x, y \in X$.

Let $\mathcal{C}$ be a coloring of arcs and regions of a given diagram $K$. Specifically, for a coloring $\mathcal{C}$, there is a coloring of regions that extend $\mathcal{C}$ as depicted in Fig. 5. Suppose that two regions $R_{1}$ and $R_{2}$ are separated by an arc colored by $y$, and the normal of the arc points from $R_{1}$ to $R_{2}$. If $R_{1}$ is colored by $x$, then $R_{2}$ receives the color $x * y$. Let $\left(x_{\tau}, y_{\tau}, z_{\tau}\right)$ (called the ordered triple of colors at a crossing $\tau$ ) be the colors near a crossing $\tau$ such that $x$ is the color of the region (called the source region) from which both orientation normals of over- and under-arcs point, $y$ is the color of the under-arc (called the source under-arc) from which the normal of the over-arc points, and $z$ is the color of the over-arc. See Fig. 5. Let $\left(x_{\tau}, y_{\tau}, z_{\tau}\right)$ be the ordered triple of colors at a crossing $\tau$. Then the weight in this case is defined by $B(\mathcal{C}, \tau)=$ $\epsilon(\tau) \phi\left(x_{\tau}, y_{\tau}, z_{\tau}\right)$. The 3 -cocycle invariant is defined in a similar way to the 2-cocycle invariant by the multiset $\Phi_{\theta}(K)=\left\{\sum_{\tau} B(\mathcal{C}, \tau) \mid \mathcal{C} \in \operatorname{Colr}_{\mathbf{X}}(\mathrm{K})\right\}$, where $\operatorname{Colr}_{\mathrm{X}}(\mathrm{K})$ denotes the set of colorings with region colors of $K$ by $X$.

If the quandle $X$ is finite, the invariant as a multiset can be written by an expression similar to those for the state-sums; if a given multiset of group elements is $\left\{\sqcup_{m_{1}} g_{1}, \ldots, \sqcup_{m_{\ell}} g_{\ell}\right\}$, then we use the polynomial notation $m_{1} u^{g_{1}}+\cdots+m_{\ell} u^{g_{\ell}}$ where $u$ is a formal symbol. For example, the multiset value of the invariant for a trefoil with the Alexander quandle $X=\mathbb{Z}_{2}\left[t, t^{-1}\right] /\left(t^{2}+t+1\right)$ with the same coefficient $A=X$ and a certain 2-cocycle is $\left\{\sqcup_{4}(1), \sqcup_{12}(t+1)\right\}$, and is denoted by $4+12 u^{(t+1)}$, where we use the convention $u^{0}=1$ and exponential rules apply.

For computing the invariants, one needs an explicit formula for cocycles. Polynomial expressions were used first in [15], and investigated closely 
including higher dimensional cocycles in [2].

\section{Boundary monochromatic colorings and the co- cycle invariants of tangles}

We use quandle cocycle invariants as obstructions to embedding tangles in knots. We first define cocycle invariants for tangles.

Definition 3.1 Let $T$ be a tangle and $X$ be a quandle. A (boundarymonochromatic) coloring $\mathcal{C}: \mathcal{A} \rightarrow X$ is a map from the set of arcs in a diagram of $T$ to $X$ satisfying the same quandle coloring condition as for knot diagrams at each crossing, such that the (four) boundary points of the tangle diagram receive the same element of $X$.

For a coloring $\mathcal{C}$ of a tangle diagram $T$, a region colorings are defined in a similar manner as in the knot case. In this case, we allow region colors to change (not necessarily colored by the same element as the one assigned to the boundary points).

Denote by $\operatorname{Col}_{x}(T)$ and $\operatorname{Col}_{X}(T)$ the set of boundary-monochromatic colorings of $T$ with the boundary color $x \in X$ and the set of all boundarymonochromatic colorings, respectively. Let $\Phi(T, x)=\sum_{C \in C_{0 l}(T)} \prod_{\tau} B(C, \tau)$. Then the cocycle invariant for a tangle $T$ is defined by $\Phi_{\phi}(T)=\sum_{x \in X} \Phi(T, x)$. The invariants with region colors are defined in a similar manner, by taking sum over all colorings of regions as well as colorings of diagrams.

It is seen in a way similar to the knot case that the number of colorings $\left|\mathrm{Col}_{X}(T)\right|$ does not depend on a choice of a diagram of $T$. Any coloring $\mathcal{C}_{1}$ of a diagram of a tangle $T$ is changed via a sequence of Reidemeister moves (with boundary points fixed) to a coloring $\mathcal{C}_{2}$ of $T$. Given two diagrams $D_{1}$ and $D_{2}$ of a tangle $T$, there is one-to-one correspondence between the set of colorings of $D_{1}$ and the set of colorings of $D_{2}$ and the cocycle invariant is well-defined.

Table 1 summarizes the tangles in the tangle table that have non-trivial boundary monochromatic colorings by some Alexander quandles. These are found by hand calculations, occasionally assisted by Maple. Specifically, variables $x_{i}, i=1,2, \ldots$, are assigned on arcs of tangle diagrams as indicated in Fig. 6, and the coloring conditions of the form $x_{k}=t x_{i}+(1-t) x_{j}$ are imposed corresponding to crossings, and the system of linear equations in $\mathbb{Z}\left[t, t^{-1}\right]$ are solved to find which Alexander quandles have non-trivial colorings. These tangles with non-trivial colorings by Alexander quandles 


\begin{tabular}{|l||l|}
\hline Quandle & Tangle colored \\
\hline $\mathbb{Z}_{p}\left[t, t^{-1}\right] /\left(t^{2}-t+1\right)$ & $6_{2}, 6_{3}, 7_{17}(\mathrm{NW}$ In, SW In $)$. \\
\hline $\mathbb{Z}_{2}\left[t, t^{-1}\right] /\left(t^{2}+t+1\right)$ & $6_{2}, 6_{3}, 7_{17}(\mathrm{NW}$ In, SW In $)$, \\
& $7_{4}(\mathrm{NW}$ In, NE In $), 7_{5}(\mathrm{NW}$ In, NE In $)$, \\
& $7_{6}(\mathrm{NW}$ In, NE In $), 7_{7}(\mathrm{NW}$ In, NE In $)$, \\
\hline$R_{3}$ & $6_{2}, 6_{3}, 7_{16}, 7_{17}$. \\
\hline$R_{5}$ & $7_{13}, 7_{18}$. \\
\hline$R_{7}$ & $7_{15}$. \\
\hline
\end{tabular}

Table 1: Tangles with non-trivial colorings

are depicted in Fig. 6. This list compares with the original list in [10] as follows. Their list starts with one 5 -crossing tangle $5_{1}$, which colors trivially by Alexander quandles and is not listed in Fig. 6. There are four $\left(6_{1}-6_{4}\right)$ 6 -crossings tangles, three of which are in our list. Thirteen out of eighteen 7 -crossing tangles are in our list.

\section{Quandle cocycle invariants as obstructions to tangle embeddings}

The quandle 2- and 3-cocycle invariants are defined for tangles in a manner similar to the knot case using the set of boundary monochromatic colorings, and denoted by $\Phi_{\phi}(T)$. We use the multiset version of the invariant.

Definition 4.1 The inclusion of multisets is denoted by $\subset_{m}$. Specifically, if an element $x$ is repeated $n$ times in a multiset, call $n$ the multiplicity of $x$, then $M \subset_{m} N$ for multisets $M, N$ means that if $x \in M$, then $x \in N$ and the multiplicity of $x$ in $M$ is less than or equal to the multiplicity of $x$ in $N$.

Theorem 4.2 Let $T$ be a tangle and $X$ a quandle. Suppose $T$ embeds in a link $L$. Then we have the inclusion $\Phi_{\phi}(T) \subset_{m} \Phi_{\phi}(L)$.

Proof. Suppose a diagram of $T$ embeds in a diagram of $L$. We continue to use $T$ and $L$ for these diagrams. For a coloring $\mathcal{C}$ of $T$, let $x$ be the color of the boundary points. Then there is a unique coloring $\mathcal{C}^{\prime}$ of $L$ such that the restriction of $\mathcal{C}^{\prime}$ on $T$ is $\mathcal{C}$ and all the $\operatorname{arcs}$ of $L$ outside of $T$ receive the color $x$. Then the contribution of $\sum_{\tau \in T} B(\mathcal{C}, \tau)$ to $\Phi_{\phi}(T)$ is equal to the contribution $\sum_{\tau \in L} B\left(\mathcal{C}^{\prime}, \tau\right)$ to $\Phi_{\phi}(L)$, and the theorem follows. The same argument works for region colors and 3 -cocycle invariants. 

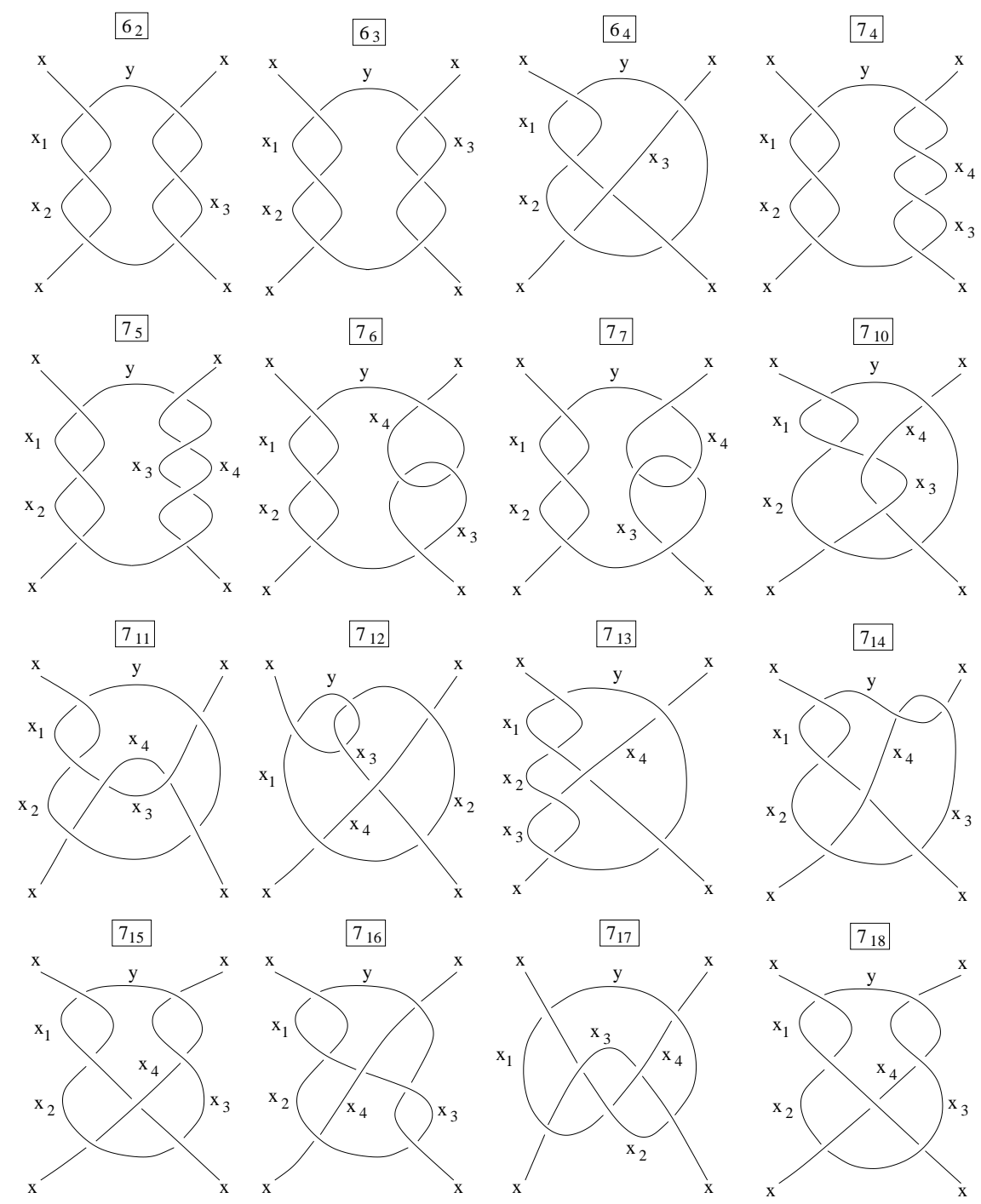

Figure 6: Tangles with non-trivial colorings by Alexander quandles 
In Table 2, a summary is presented for the tangles that color non-trivially by Alexander quandles. In the left column of the table, the tangles that appear in Table 1 are listed. In the middle column, knots that we found to embed a given tangle are listed. The third column lists the knots for which we could not exclude the possibility of embedding of the given tangle using cocycle invariants. The tangles are specified by the notation $T\left(6_{2}\right)$, for example, for the tangle numbered $6_{2}$ to distinguish them from knots. We note that there are 84 knots in the table up to (including) 9-crossing knots. For the tangle $T\left(6_{3}\right)$, for example, all except 3 out of 84 are detected by the cocycle invariants that they do not embed the tangle. It is checked by hand that these remaining three do embed it.

To demonstrate how we obtain these results, we state and prove the following.

Proposition 4.3 The tangle $T\left(6_{2}\right)$ with the orientation of the $N W$ arc inward and the $S W$ arc outward does not embed in the knots in the table up to 9 crossings except, possibly, for $8_{18}, 9_{29}, 9_{38}$.

Proof. With this orientation, the tangle is of the form of two copies of the mirror of the trefoil, and is colored non-trivially by the quandle $Z_{p}[t] /\left(t^{2}-\right.$ $t+1)$.

We exhibit a method to determine the invariant from the table in [21. For $p=2$, the table of quandle cocycle invariants in [21] gives $16+48 u^{t}$ as the invariant for trefoil with the 3-cocycle $\phi(x, y, z)=(x-y)(y-z)^{2}$. This implies that any non-trivial coloring contributes $t$ to the invariant. Its mirror has the same property. With two copies, any non-trivial coloring of the tangle contributes $2 t=0$ when $p=2$. Hence the invariant value of the tangle is 64 . From the table this does not embed in knots up to 9 crossings except for the following possibilities : $8_{5}, 8_{10}, 8_{15}, 8_{18}$, $8_{19}, 8_{20}, 8_{21}, 9_{16}, 9_{22}, 9_{24}, 9_{25}, 9_{28}, 9_{29}, 9_{30}, 9_{36}, 9_{38}, 9_{39}, 9_{40}, 9_{41}, 9_{42}, 9_{43}, 9_{44}$, $9_{45}, 9_{49}$.

For $p=3$, the invariant table gives $243+486 u^{(2 t+2)}$ as the invariant for trefoil. This implies that 486 non-trivial colorings contributes $2 t+2$ to the invariant. Its mirror contributes $t+1$. With two copies, 486 non-trivial colorings of the tangle contributes $2 t+2$. Hence the invariant value of the tangle is $243+486 u^{(2 t+2)}$. From the table this does not embed in knots up to 9 crossings except for: $3_{1}, 8_{18}, 9_{2}, 9_{4}, 9_{29}, 9_{34}, 9_{38}$.

For $p=5$, the table gives

$$
625+3750 u^{(t+3)}+3750 u^{(4 t+2)}+3750 u^{(3 t+4)}+3750 u^{(2 t+1)}
$$




\begin{tabular}{|c|c|c|}
\hline Tangle & Embeds in: & May embed in: \\
\hline 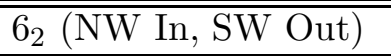 & $\bar{~}\left(8_{5}\right)^{*}=N\left(T\left(6_{2}\right)+R(-2)\right)$ & $8_{18}, 9_{29}, 9_{38}$. \\
\hline $6_{2}(\mathrm{NW}$ In, SW In) & $\left(3_{1}\right)=N\left(T\left(6_{2}\right)+R(-1)\right)$ & $\begin{array}{l}3_{1}, 7_{4}, 7_{7}, 8_{18}, 9_{10}, 9_{29} \\
9_{35}, 9_{37}, 9_{38}, 9_{46}, 9_{48}\end{array}$ \\
\hline $66_{3}$ (NW In, SW Out) & $\begin{array}{l}\left(8_{10}\right)=N\left(T\left(6_{3}\right)+R(2,1)\right)^{*} \\
\left(8_{20}\right)=N\left(T\left(6_{3}\right)+R(2)\right)^{*} \\
\left(9_{24}\right)=N\left(T\left(6_{3}\right)+R(2,2)\right)^{*}\end{array}$ & $8_{10}, 8_{20}, 9_{24}$. \\
\hline $7_{4}(\mathrm{NW}$ In, NE In) & $\left(4_{1}\right)=\left(N\left(T\left(7_{4}\right)+R(-1)\right)\right.$ & $\begin{array}{l}3_{1}, 4_{1}, 7_{2}, 7_{3}, 8_{1}, 8_{4}, 8_{11}, \\
8_{13}, 8_{18}, 9_{1}, 9_{6}, 9_{12}, 9_{13}, \\
9_{14}, 9_{21}, 9_{23}, 9_{35}, 9_{37}, 9_{40}\end{array}$ \\
\hline $7_{5}(\mathrm{NW}$ In, NE In) & $\left(7_{3}\right)^{*}=N\left(T\left(7_{5}\right)+R(-1)\right)$ & Same as $7_{4}(\mathrm{NW}$ In, NE In). \\
\hline $7_{6}(\mathrm{NW}$ In, NE In) & & $\begin{array}{l}8_{5}, 8_{10}, 8_{15}, 8_{18}-8_{21}, 9_{16} \\
9_{22}, 9_{24}, 9_{25}, 9_{28}-9_{30}, 9_{36} \\
9_{38}, 9_{39}, 9_{41}-9_{45}, 9_{49}\end{array}$ \\
\hline $7_{7}(\mathrm{NW}$ In, NE In) & & Same as $7_{6}(\mathrm{NW}$ In, NE In). \\
\hline $7_{13}$ (NW In, NE Out) & $\begin{array}{l}\left(7_{4}\right)=N\left(T\left(7_{13}\right)\right) \\
\left(8_{16}\right)=N\left(T\left(7_{13}\right)+R(1)\right) \\
\left(9_{39}\right)=N\left(T\left(7_{13}\right)+R(1,1)\right) \\
\left(9_{49}\right)=N\left(T\left(7_{13}\right)\right. \\
\quad+R(-1,-1)) \\
\quad\end{array}$ & $4_{1}, 7_{4}, 9_{24}, 9_{37}, 9_{39}, 9_{40}, 9_{49}$. \\
\hline $7_{15}(\mathrm{NW}$ In, SW In) & $\left(5_{2}\right)=N\left(T\left(7_{15}\right)+R(-1)\right)$ & $5_{2}, 8_{16}, 9_{41}, 9_{42}$. \\
\hline $7_{15}$ (NW In, SW Out) & $\begin{array}{l}\left(7_{7}\right)=D\left(T\left(7_{15}\right)\right) \\
\left(9_{41}\right)=N\left(T\left(7_{15}\right)+R(2)\right)\end{array}$ & $7_{1}, 7_{7}, 8_{5}, 9_{4}, 9_{12}, 9_{41}$. \\
\hline $7_{16}(\mathrm{NW}$ In, NE In) & $\left(7_{7}\right)^{*}=D\left(T\left(7_{16}\right)\right)$ & $\begin{array}{l}8_{5}, 8_{15}, 8_{18}, 8_{19}, 8_{21}, 9_{2}, 9_{4}, \\
9_{11}, 9_{15}, 9_{16}, 9_{28}, 9_{34}, 9_{37} \\
9_{40}, 9_{46}, 9_{47}\end{array}$ \\
\hline $7_{16}(\mathrm{NW}$ In, NE Out) & $\left(7_{4}\right)=N\left(T\left(7_{16}\right)\right)$ & Same as $6_{2}(\mathrm{NW}$ In, SW In). \\
\hline $7_{17}(\mathrm{NW}$ In, SW In) & $\left(8_{18}\right)=N\left(T\left(7_{17}\right)+R(1)\right)$ & $8_{18}, 9_{40}$ \\
\hline $7_{17}$ (NW In, SW Out) & & Same as $7_{16}(\mathrm{NW}$ In, NE In). \\
\hline $7_{18}(\mathrm{NW}$ In, SW In) & $\left(8_{21}\right)=N\left(T\left(7_{18}\right)+R(1)\right)$ & $\begin{array}{l}5_{1}, 8_{18}, 8_{21}, 9_{2}, 9_{12}, 9_{23}, 9_{31} \\
9_{40}, 9_{49}\end{array}$ \\
\hline $7_{18}(\mathrm{NW}$ In, SW Out) & $\left(5_{1}\right)=D\left(T\left(7_{18}\right)\right)$ & Same as $7_{18}(\mathrm{NW}$ In, SW In). \\
\hline
\end{tabular}

Table 2: Summary of the results 
as the invariant for the trefoil. As in the previous cases, the tangle has the invariant value

$$
625+3750 u^{(3 t+4)}+3750 u^{(2 t+1)}+3750 u^{(4 t+2)}+3750 u^{(t+3)}
$$

(for example, for the contribution $t+3$ of trefoil, the mirror contributes $4 t+2$, its double contributes $3 t+4)$. From the table this does not embed in knots up to 9 crossings except for: $3_{1}, 8_{3}, 8_{5}, 8_{11}, 8_{15}, 8_{18}, 8_{19}, 8_{21}, 9_{1}, 9_{5}, 9_{6}, 9_{16}, 9_{19}$, $9_{23}, 9_{28}, 9_{29}, 9_{38}, 9_{40}$.

For $p=7$, the trefoil has 117649 as the invariant value, and so does the tangle. From the table this does not embed in knots up to 9 crossings except for: $3_{1}, 8_{5}, 8_{10}, 8_{11}, 8_{15}, 8_{18}, 8_{19}, 8_{20}, 8_{21}, 9_{1}, 9_{6}, 9_{16}, 9_{23}, 9_{28}, 9_{29}, 9_{38}, 9_{40}$.

From all these information combined, this tangle does not embed in knots up to 9 crossings except for the only possibilities of $8_{18}, 9_{29}, 9_{38}$.

We have not been able to determine whether the tangle $T\left(6_{2}\right)$ actually embeds in these three knots that the invariant failed to exclude. In the next example, however, we were able to determine completely the embedding problem up to 9 crossings.

Proposition 4.4 The knots in the table up to 9 crossings in which the tangle $T\left(6_{3}\right)$ embeds are exactly $8_{10}, 8_{20}, 9_{24}$. Here, the orientation of the tangle is such that the end point $N W$ is oriented inward and the $S W$ end point is oriented outward.

Proof. The tangle $T\left(6_{3}\right)$ is written as the addition $R(3)+R(-3)$. Hence it is colored non-trivially by $Z_{p}[t] /\left(t^{2}-t+1\right)$ for any $p \in \mathbb{Z}$ (we use only primes), as well as the dihedral quandle $R_{3}$. For the quandle $Z_{p}[t] /\left(t^{2}-t+1\right)$ we used the 3 -cocycle $f(x, y, z)=(x-y)(y-z)^{p}$. The colors of the source region for these two copies of the trefoil diagrams $(R(3)$ and $R(-3))$ coincide. The signs of the crossings are opposite. Hence the invariant is trivial, $\left(p^{2}\right)^{3}$ copies of 0 , for $Z_{p}[t] /\left(t^{2}-t+1\right)$. For $p=5$, in particular, from the calculations in [21], Theorem 4.2 implies that this tangle may embed, among knots in the table up to 9 crossings, only in: $8_{10}, 8_{12}, 8_{18}, 8_{20}, 9_{24}$. The invariant with $R_{3}$ further excludes $8_{12}$ and $8_{18}$. Therefore the tangle may embed only in $8_{10}, 8_{20}$, and $9_{24}$.

On the other hand, it is seen that

$$
\begin{aligned}
& \left(8_{10}\right)=N\left(T\left(6_{3}\right)+R(2,1)\right)^{*}, \\
& \left(8_{20}\right)=N\left(T\left(6_{3}\right)+R(2)\right)^{*}, \\
& \left(9_{24}\right)=N\left(T\left(6_{3}\right)+R(2,2)\right)^{*},
\end{aligned}
$$


where $K^{*}$ denotes the mirror image of a knot $K$, and $R$ denotes the rational tangles. Note that this tangle $T\left(6_{3}\right)$ is equivalent to its mirror. Therefore we have shown that the tangle $T\left(6_{3}\right)$ does indeed embed in these three knots.

Remark 4.5 In general the orientation needs to be specified to define the quandle cocycle invariants. (In our case only the dihedral quandles can be used for the invariant without specifying the orientations [20].) Furthermore, the mirror images of a given knot in the table may be different. Thus all of our results are stated for oriented tangles and oriented knots, and do not include their mirror images. Our convention for specifying orientations of tangles are already explained. For knots in the table, we used Livingston's table [13], which includes particular choices of mirrors if a knot is not amphicheiral. For the orientations, we used the braid form in [13], for our calculations, so that the orientations are specified by downward orientations of the braids.

\section{$5 \quad$ Embedding disjoint tangles}

In this section we discuss embeddings of disjoint union of tangles in knots. We prove a theorem that will be used as obstruction to embedding disjoint union of tangles and give some examples.

Let $C=\sum_{i=1}^{k} m_{i} u^{c_{i}}, D=\sum_{j=1}^{\ell} n_{j} u^{d_{j}}$ be polynomial expressions of multisets values of the invariants, where $m_{i}, n_{j} \in \mathbb{Z}_{+}, c_{i}, d_{j} \in A$, where $A$ is the coefficient abelian group. Then we define $C \times D=\sum_{i, j} m_{i} n_{j} u^{c_{i}+d_{j}}$. Let $|X|$ denote the number of elements of a quandle $X$.

The quandle cocycle invariants are defined for disjoint union of tangles $T_{1} \sqcup \cdots \sqcup T_{k}$ in a manner similar to tangles requiring that all the boundary points of $T_{1}, \ldots, T_{k}$ receive the same color. Let $\phi$ be a 2-cocycle of a quandle $X$ and we define

$$
\Phi_{\phi}\left(T_{1} \sqcup \cdots \sqcup T_{k}\right)=\sum_{x_{j} \in X} \prod_{i=1}^{k} \Phi_{\phi}\left(T_{i}, x_{j}\right) .
$$

Proposition 5.1 Let $\phi$ be a 2-cocycle. Let $T_{1}, \ldots, T_{k}$ be a disjoint union of tangles such that for all $i=1, \ldots, k$, the condition $\Phi_{\phi}\left(T_{i}, x\right)=\Phi_{\phi}\left(T_{i}, y\right)$ holds for all $x, y \in X$. Then we have

$$
\Phi_{\phi}\left(T_{1} \sqcup \cdots \sqcup T_{k}\right)=\frac{1}{|X|^{k-1}} \Phi_{\phi}\left(T_{1}\right) \times \cdots \times \Phi_{\phi}\left(T_{k}\right) .
$$


Furthermore if a disjoint union of $T_{1}, \ldots, T_{k}$ embed in a link $L$, then

$$
\Phi_{\phi}\left(T_{1} \sqcup \cdots \sqcup T_{k}\right) \subset_{m} \Phi_{\phi}(L) .
$$

Proof. We compute

$$
\Phi_{\phi}\left(T_{1} \sqcup \cdots \sqcup T_{k}\right)=\sum_{x_{j} \in X} \prod_{i=1}^{k} \Phi\left(T_{i}, x_{j}\right)=|X| \prod_{i=1}^{k} \Phi\left(T_{i}, x\right)
$$

for any fixed $x \in X$ since $\Phi\left(T_{i}, x\right)=\Phi\left(T_{i}, y\right)$ for all $x, y \in X$. The condition also implies that $\Phi\left(T_{i}, x\right)=\frac{1}{|X|} \Phi_{\phi}\left(T_{i}\right)$ for all $i=1, \ldots, k$. Hence

$$
\Phi_{\phi}\left(T_{1} \sqcup \cdots \sqcup T_{k}\right)=\frac{1}{|X|^{k-1}} \Phi_{\phi}\left(T_{1}\right) \times \cdots \times \Phi_{\phi}\left(T_{k}\right) .
$$

Thus by the same argument as the proof of Theorem 4.2, if $T_{1} \sqcup \cdots \sqcup T_{k}$ embeds in a link $L$, we have

$$
\frac{1}{|X|^{k-1}} \Phi_{\phi}\left(T_{1}\right) \times \Phi_{\phi}\left(T_{2}\right) \times \cdots \times \Phi_{\phi}\left(T_{k}\right) \subset_{m} \Phi_{\phi}(L) .
$$

Example 5.2 For the following examples, we consider the quandle $X=$ $\mathbb{Z}_{2}\left[t, t^{-1}\right] /\left(t^{2}+t+1\right)$, and the 2-cocycle $f(x, y)=(x-y)^{2} y$. The invariant values for this quandle are available in [21] (here we used knots up to 9 -crossings). It is seen that the following tangles satisfy the condition required in Proposition 5.1 by direct calculations. Alternatively, either of the triviality of the invariant, or the property that only the trivial colorings make trivial contributions to the invariant, implies the condition required.

(a) We compute $\Phi_{f}\left(T\left(6_{2}\right) \sqcup T\left(6_{2}\right)\right)=\frac{1}{4}(16 \times 16)=64$ (Proposition 4.3). Using [21] we compare this invariant to the cocycle invariant of knots in the knot table, and conclude that $T\left(6_{2}\right) \sqcup T\left(6_{2}\right)$ does not embed in any knot in the knot table up to 9 crossings.

The invariant value of $T\left(6_{3}\right)$ is $\Phi_{f}\left(T\left(6_{3}\right)\right)=16$ by an argument similar to those used for 3-cocycles in the proof of Proposition 4.4. By theorem 5.1 $\Phi_{f}\left(T\left(6_{3}\right) \sqcup T\left(6_{3}\right)\right)=\frac{1}{4}(16 \times 16)=64$. Hence $T\left(6_{3}\right) \sqcup T\left(6_{3}\right)$ does not embed in any knot in the knot table up to 9 crossings.

The disjoint union $T\left(6_{2}\right) \sqcup T\left(6_{3}\right)$ also has the same invariant value 64 , hence the same conclusion holds.

(b) The invariant of the tangle $T\left(7_{5}\right)$ with orientation (NW In, NE In) is $\Phi_{f}\left(T\left(7_{5}\right)\right)=4+12 u^{(t+1)}$. This can be seen from the fact that $T\left(7_{5}\right)$ 
embeds in the knot $\left(7_{3}\right)^{*}$ and the number of colorings by this quandle is the same for $T\left(7_{5}\right)$ and $\left(7_{3}\right)^{*}$, so that by an argument similar to the proof of Proposition 4.3, the tangle has the same invariant value as $\left(7_{3}\right)^{*}$ (see [21]). Hence by Theorem 5.1 we obtain

$$
\Phi_{f}\left(T\left(7_{5}\right) \sqcup T\left(7_{5}\right)\right)=\frac{1}{4}\left(4+12 u^{(t+1)}\right)^{2}=40+24 u^{(t+1)} .
$$

Using [21, we compare this invariant to the cocycle invariant of knots in the table, and we conclude that $T\left(7_{5}\right) \sqcup T\left(7_{5}\right)$ does not embed in any knot in the table up to 9 crossings.

(c) Again by Theorem 5.1,

$$
\Phi_{f}\left(T\left(6_{2}\right) \sqcup T\left(7_{5}\right)\right)=\frac{1}{4} 16\left(4+12 u^{(t+1)}\right)=16+48 u^{(t+1)} .
$$

We find that $T\left(6_{2}\right) \sqcup T\left(7_{5}\right)$ does not embed in any knot in the knot table up to 9 crossings with possible exceptions of $8_{18}$ and $9_{40}$.

Since the invariant value for $T\left(6_{3}\right) \sqcup T\left(7_{5}\right)$ is the same as $T\left(6_{2}\right) \sqcup T\left(7_{5}\right)$, we obtain the same conclusion.

Let $\psi$ be a 3-cocycle of a quandle $X$ with coefficient group $A$. Denote by $\Phi_{\psi}(T, x, s)$ the 3-cocycle invariant with the boundary color $x \in X$ and the color of the leftmost region $s \in X$. Then the 3-cocycle invariant for disjoint union of tangles $\sqcup_{i=1}^{k} T_{i}$ is defined if $T_{i}$ satisfy the condition $\Phi_{\psi}\left(T_{i}, x, s\right)=$ $\Phi_{\psi}\left(T_{i}, x^{\prime}, s^{\prime}\right)$ for all $x, x^{\prime}, s, s^{\prime} \in X$ for all $i=1, \ldots, k$, and is defined in this case by

$$
\Phi_{\psi}\left(T_{1} \sqcup \cdots \sqcup T_{k}\right)=\sum_{x_{j} \in X, s \in X} \prod_{i=1}^{k} \Phi_{\phi}\left(T_{i}, x_{j}, s\right)=|X| \sum_{x_{j} \in X} \prod_{i=1}^{k} \Phi_{\phi}\left(T_{i}, x_{j}, s\right)
$$

for a fixed $s \in X$ (and the invariant does not depend on this choice of $s$ ). Note that the invariant does not depend on a fixed region color $s$ because of the above assumption. Then the same argument as the proof of the preceding theorem can be applied to show the following.

Proposition 5.3 Let $\psi$ be a 3 -cocycle. Let $T_{1}, \ldots, T_{k}$ be a disjoint union of tangles such that for all $i=1, \ldots, k$, the condition $\Phi_{\psi}\left(T_{i}, x, s\right)=\Phi_{\psi}\left(T_{i}, x^{\prime}, s^{\prime}\right)$ holds for all $x, x^{\prime}, s, s^{\prime} \in X$. Then we have

$$
\Phi_{\psi}\left(T_{1} \sqcup \cdots \sqcup T_{k}\right)=\frac{1}{|X|^{2(k-1)}} \Phi_{\psi}\left(T_{1}\right) \times \cdots \times \Phi_{\psi}\left(T_{k}\right) .
$$


Furthermore if a disjoint union of $T_{1}, \ldots, T_{k}$ embed in a link $L$, then

$$
\Phi_{\psi}\left(T_{1} \sqcup \cdots \sqcup T_{k}\right) \subset_{m} \Phi_{\psi}(L) .
$$

Example 5.4 We used dihedral quandles $R_{p}$ with Mochizuki's cocycle [15]

$$
\psi(x, y, z)=(1 / p)(x-y)\left[\left(2 z^{p}-y^{p}\right)-(2 z-y)^{p}\right] \quad(\bmod p) .
$$

The invariant values are available in [21] up to 12-crossing knots for $p=3,5$. By arguments similar to those in Example 5.2, it is seen that the following tangles satisfy the condition required in Proposition 5.3 .

(a) For the dihedral quandle $R_{3}$ and the Mochizuki's 3-cocycle $\psi$, the tangle $T\left(6_{2}\right)$ satisfies the condition in Theorem [5.3. This is because $T\left(6_{2}\right)$ is the sum of two copies of part of trefoil diagrams, and the trefoil has the property that any non-trivial coloring gives the same non-trivial contribution to the cocycle invariant. Since $\Phi_{\psi}\left(T\left(6_{2}\right)\right)=9(1+2 u)$, by Theorem 5.1 we obtain $\Phi_{\psi}\left(T\left(6_{2}\right) \sqcup T\left(6_{2}\right)\right)=\frac{1}{3^{2}} 81\left(1+4 u+4 u^{2}\right)=9+36 u+36 u^{2}$. Using [21] we compare this invariant to the cocycle invariant of knots in the knot table, and we find that $T\left(6_{2}\right) \sqcup T\left(6_{2}\right)$ does not embed in any knot in the knot table up to 11 crossings (there are 801) except, possibly, $8_{18}$ and $11 a_{314}$. From the invariant value, the number of colorings of $T\left(6_{2}\right) \sqcup T\left(6_{2}\right)$ is 81 , and among 801 knots in the table up to 11 crossings, there are 40 with at least 81 colorings. Hence the number of colorings alone can exclude all but 40 knots, but the cocycle invariant is able to exclude all but 2 .

(b) $\Phi_{\psi}\left(T\left(6_{3}\right) \sqcup T\left(6_{3}\right)\right)=\frac{1}{3^{2}} 3^{3} 3^{3}=81$ with $R_{3}$, and $T\left(6_{3}\right) \sqcup T\left(6_{3}\right)$ does not embed in any knot in the knot table up to 11 crossings, except possibly $10_{99}$, hence the cocycle invariant excludes all 801 knots but one.

(c) For the quandle $R_{5}$ and tangles $T\left(7_{13}\right)$ and $T\left(7_{18}\right)$ both have invariant $25\left(1+2 u+2 u^{3}\right)$, hence $T\left(7_{13}\right) \sqcup T\left(7_{18}\right)$ has invariant $25\left(5+u+4 u^{2}+2 u^{3}+4 u^{4}\right)$. Thus it does not embed in any knot in the knot table up to 11 crossings, except possibly $10_{103}, 10_{155}, 11 a_{317}, 11 n_{148}$.

Acknowledgments. We would like to thank J.S. Carter and S. Satoh for valuable conversations.

\section{References}

[1] C. Adams, The knot book: An elementary introduction to the mathematical theory of knots, American Mathematical Society, Providence, RI, 1994, 2004 
[2] K. Ameur, Polynomial quandle cocycles, their knot invariants and applications, Ph.D. dissertation, University of South Florida (2007).

[3] E. Brieskorn, Automorphic sets and singularities, in "Contemporary math." 78 (1988) 45-115.

[4] J.S. Carter; D. Jelsovsky; S. Kamada; L. Langford; M. Saito, Quandle cohomology and state-sum invariants of knotted curves and surfaces, Trans. Amer. Math. Soc. 355 (2003) 3947-3989.

[5] J-W. Chung; X-S. Lin, On n-punctured ball tangles, J. Knot Theory Ramifications 15 (2006) 715-748.

[6] I.K. Darcy; J. Navarra-Madsen, Coloring n-String Tangles, Preprint, arXive:math.GT/0609587.

[7] C. Ernst; D.W. Sumners, A calculus for rational tangles: applications to DNA recombination, Math. Proc. Cambridge Philos. Soc. 108 (1990) 489-515.

[8] R. Fenn; C. Rourke, Racks and links in codimension two, J. Knot Theory Ramifications 1 (1992) 343-406.

[9] D. Joyce, A classifying invariant of knots, the knot quandle, J. Pure Appl. Alg. 23 (1982) 37-65.

[10] T. Kanenobu; H. Saito; S. Satoh, Tangles with up to seven crossings, Interdisciplinary Infomation Sciences 9 (2003) 127-140.

[11] D.A. Krebes, An obstruction to embedding 4-tangles in links, J. Knot Theory Ramifications 8 (1999) 321-352.

[12] D.A. Krebes; D.S. Silver; S.G. Williams, Persistent invariants of tangles, J. Knot Theory Ramifications 9 (2000) 471-477.

[13] C. Livingston, Table of knot invariants, http://www.indiana.edu/ knotinfo/.

[14] S. Matveev, Distributive groupoids in knot theory (Russian), Math. USSR-Sbornik 47 (1982) 73-83.

[15] T. Mochizuki, Some calculations of cohomology groups of finite Alexander quandles, J. Pure Appl. Algebra 179 (2003) 287-330.

[16] K. Murasugi, Knot theory and its applications, Birkhauser, Boston Inc, Boston, MA 1996.

[17] M. Niebrzydowski, On colored quandle longitudes and its applications to tangle embeddings and virtual knots, J. Knot Theory Ramifications $\mathbf{1 5}$ (2006) 1049-1059.

[18] J.H. Przytycki; D.S. Silver; S.G. Williams, 3-Manifolds, tangles and persistent invariants, Math. Proc. Cambridge Philos. Soc. 139 (2005) 291-306. 
[19] D. Ruberman, Embedding tangles in links, J. Knot Theory Ramifications 9 (2000) 523-530.

[20] S. Satoh, On chirality of Suzuki's $\theta_{n}$-curves, Preprint.

[21] C.Smudde, Computer program, available at http://shell.cas.usf.edu/quandle/. 